\title{
Everolimus and prednisolone alone as maintenance immunosuppressive treatment in a patient receiving chemotherapy for metastatic cancer colon
}

\section{M.K.Habeeb" ${ }^{192}$, M.F.Ramzy ${ }^{1}$, A.Halawa ${ }^{2}$ and A.Sharma}

\section{Introduction}

Although new immunosuppressive medications have dramatically improved the outcomes of kidney transplantation, they also have several adverse effects like infections, cardiovascular accidents and malignancies [1]. Among transplant recipients, malignancy is considered one of the leading causes of death along with cardiovascular accidents and infections. The incidence of gastrointestinal malignancies including $\mathrm{CRC}$ is higher (about 2 times) in transplant recipients in comparison with general population [2].

\section{The Case}

A 64 year old male patient with chronic kidney disease due to renal amyloidosis secondary to familial Mediterranean fever with no family history of any cancer, underwent living non-related kidney transplantation (1-11 mismatch). On discharge he was put on triple therapy tacrolimus, mycophenolic acid and prednisolone. 7 months after transplantation he started to have left sided colicky abdominal pain and altered bowel habits mainly constipation, abdominal ultrasound showed mural thickening

Thickening of colon by US

about $17 \mathrm{~mm}$ of the descending and sigmoid colon, CT scan revealed the same findings.

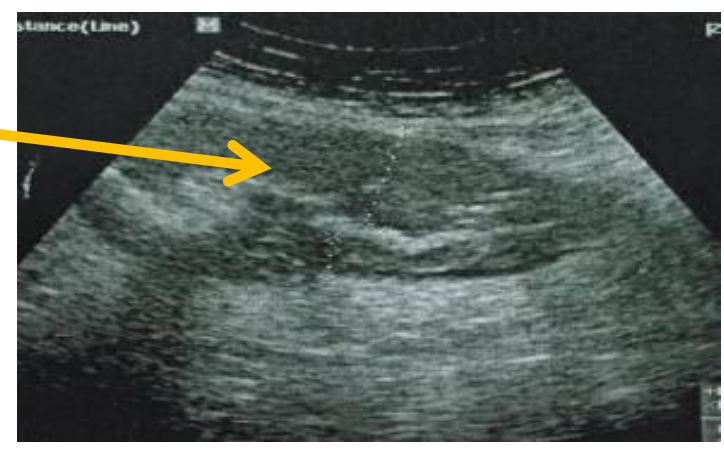

Extended left hemicolectomy was done. Pathological analysis of the specimen revealed infiltrating adenocarcinoma grade II stage B, PET scan showed liver metastases, the immunosuppressant medications was modified as follow everolimus $0.75 \mathrm{mg}$ twice daily and prednisolone $20 \mathrm{mg}$ which reduced gradually to $10 \mathrm{mg}$. He received chemotherapy in the form of FolFox 6 doses after which liver deposits completely disappeared, then reappeared again after 10 months as discovered by PET scan. Now he is on another chemotherapy protocol with his creatitine level $1.1 \mathrm{mg} / \mathrm{dl}$.
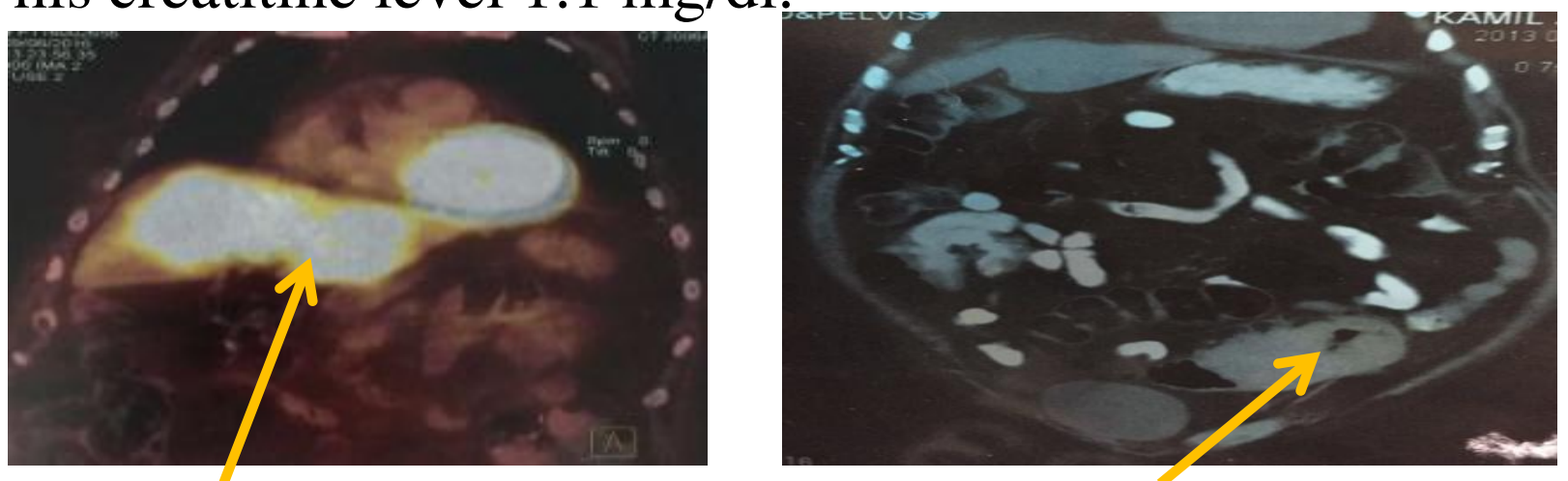

Liver metastases by

Colonic mass by CT scan

PET scan

1- Misr kidney center, Cairo.

2- Institute of teaching and live science, university of Liverpool.

\section{Introduction}

Malignancy is considered one of the most important contributors to recipient's morbidity and mortality and around one third of deaths with functioning renal allograft are due to cancer [3]. Different studies discussed the incidence of colorectal carcinoma after kidney transplantation with some controversies in their results. For example, Kasiske et al. concluded that, there is two-fold higher risk of CRC development in the first year after transplantation, which increases to 2.2 times higher risk after the third year compared with the general population [4]. On the other hand, Saidi et al. reported no increased risk of colorectal cancer among transplant recipients and noted the need for further evaluation of this issue [5].

In general the prognosis of recipients who develop CRC after kidney transplantation are poor as a result of delayed diagnosis and tendency of CRC to be biologically more aggressive in transplant. It is well known that, the duration and intensity of immunosuppressive medications are directly related to the like hood of developing malignancy posttransplantion, the more intense and prolonged immunosuppression is associated with increased risk of developing malignancies [6].

\section{Conclusions}

screening program in both pre or post-operative periods for early detection of $\mathrm{CRC}$ is needed. Minimization of immunosuppressants is usually indicated. In our case the patient has been kept only everolimus and prednisolone for 36 months till now with good graft function (serum creatinine level $1.1 \mathrm{mg} / \mathrm{dL}$ ) without any rejection episode. Even though this regimen is successfully used in our patient, larger studies are still needed to demonstrate its efficacy and drawbacks.

\section{References}

1- Stratta P, Morellini V, Musseti C, et al. Malignancy after kidney transplantation:results of 400 patients from a single center. Clin Transplant 2008; 22(4): 424.

2- Andres A. Cancer incidence after immunosuppressive treatment following kidney transplantation. Crit Rev Oncol Hematol 2005 56(1): 71-85.

3- Kauffman HM, Cherikh WS, McBride MA, Cheng Y, Hanto DW. Post-transplant de novo malignancies in renal transplant recipients: the past and present. Transpl Int 2006; 19:607-620.

4- Kasiske BL, Snyder JJ, Gilbertson DT, Wang C. Cancer after kidney transplantation in the United States. Am J Transplant 2004; 4: 905-913.

5- R. F. Saidi, P. S. Dudrick, and M. H. Goldman. Colorectal cancer after renal transplantation. Transplantation Proceedings2003; 35(4): 1410-1412

6- Man KJ, Dong JJ, Soo JK, Kyu HH, et al. Chronologically different incidences of post-transplant malignancies in renal transplant recipients: single center experience. Transpl Int 2009; 22: 644-653. 